\title{
Reducing Perceptual Interference Improves Visual Discrimination in Mild Cognitive Impairment: Implications for a Model of Perirhinal Cortex Function
}

\author{
Rachel N. Newsome, ${ }^{1 *}$ Audrey Duarte, ${ }^{2}$ and Morgan D. Barense ${ }^{1,3}$
}

\begin{abstract}
Memory loss resulting from damage to the medial temporal lobes (MTL) is traditionally considered to reflect damage to a dedicated, exclusive memory system. Recent work, however, has suggested that damage to one MTL structure, the perirhinal cortex (PRC), compromises complex object representations that are necessary for both memory and perception. These representations are thought to be critical in shielding against the interference caused by a stream of visually similar input. In this study, we administered a complex object discrimination task to two memory-impaired populations thought to have brain damage that includes the PRC [patients diagnosed with amnestic mild cognitive impairment $(\mathrm{MCl})$, and older adults at risk for $\mathrm{MCI}$ ], as well as agematched controls. Importantly, we carefully manipulated the level of interference: in the High Interference condition, participants completed a block of consecutive perceptually similar complex object discriminations, whereas in the Low Interference condition, we interspersed perceptually dissimilar objects such that there was less buildup of visual interference. We found that both memory-impaired populations were impaired on the High Interference condition compared with controls, but critically, by reducing the degree of perceptual interference, we were largely able to improve their performance. These findings, when taken together with convergent evidence from animals with selective PRC lesions and amnesic patients with focal damage to the PRC, provide support for a representational-hierarchical model of PRC function and suggest that memory loss following PRC damage may reflect a heightened vulnerability to perceptual interference. $\odot 2012$ Wiley Periodicals, Inc.
\end{abstract}

KEY WORDS: perirhinal cortex; medial temporal lobe; Alzheimer's disease; mild cognitive impairment; perception; visual discrimination; interference

\section{INTRODUCTION}

Mild cognitive impairment (MCI) is a condition that often precedes diagnosis of Alzheimer's disease, and is usually associated with memory impairments. MCI patients have incipient damage to key brain structures known to be vital for memory: namely, the medial temporal lobes

\footnotetext{
${ }^{1}$ Department of Psychology, University of Toronto, Toronto, Ontario; ${ }^{2}$ School of Psychology, Georgia Institute of Technology, Atlanta, Georgia; ${ }^{3}$ Rotman Research Institute, Toronto, Ontario

Additional Supporting Information may be found in the online version of this article.

Grant sponsor: CIHR; Grant number: MOP-115148 (MDB); Grant sponsor: Emory Alzheimer's Disease Research Center; Grant number: 2P50AG025688-06.

*Correspondence to: Rachel N. Newsome, 100 St. George Street, Toronto, ON M5S 3G3, Canada. E-mail: rachel.newsome@utoronto.ca Accepted for publication 3 August 2012

DOI 10.1002/hipo.22071

Published online in Wiley Online Library (wileyonlinelibrary.com).
}

(MTL) (e.g., Loewenstein et al., 2009; SchmidtWilcke et al., 2009). Traditionally, the MTL has been thought to exclusively support long-term declarative memory, our conscious memory for events (e.g., Squire and Zola-Morgan, 1991; Eichenbaum and Cohen, 2001). According to this theory, the primary function of the MTL is in memory and not in other aspects of cognition, such as object perception (Clark et al., 2011; Kim et al., 2011; Squire and Wixted, 2011). However, recent reports have challenged this prevailing view that the mechanisms underlying memory and perception are anatomically segregated, suggesting instead that the MTL-in particular the perirhinal cortex (PRC) - is not only important for memory but also essential for certain forms of perception (e.g., Buckley et al., 2001; Bussey and Saksida, 2002; Lee et al., 2005a; Bartko et al., 2007b; O'Neil et al., 2009; Barense et al., 2010b; Lee and Rudebeck, 2010; Barense et al., 2011a,b; Murray and Wise, 2012). These findings have led to a representational-hierarchical account of PRC function (Fig. 1), which holds that apparently distinct mnemonic and perceptual functions may arise from common representations and computational mechanisms (Murray and Bussey, 1999; Cowell et al., 2010b; Graham et al., 2010; Saksida and Bussey, 2010; Cowell, 2012). More specifically, recent studies in humans (Barense et al., 2005; Lee et al., 2005b; Barense et al., 2007; Devlin and Price, 2007; O'Neil et al., 2011), monkeys (Bussey et al., 2002; Bussey et al., 2003; Burke et al., 2011), and rats (Bartko et al., 2007a; Burke et al., 2010; McTighe et al., 2010) suggest that the PRC is necessary for processing the complex conjunctions of features comprising objects, during both memory and perceptual tasks. This property of visual discriminations has been termed "feature ambiguity," meaning that the task is not solvable using single features alone but instead requires a complex conjunction of object features. These findings suggest that the PRC should be considered an extension of the representational hierarchy within the ventral visual stream. When an object is viewed, low-level features are represented in early posterior regions of the ventral visual stream, whereas conjunctions of these low-level features are represented in more anterior regions (e.g., Desimone and Ungerleider, 1989; Tanaka, 1996; Riesenhuber and Poggio, 1999; Martinovic et al., 2008). According 

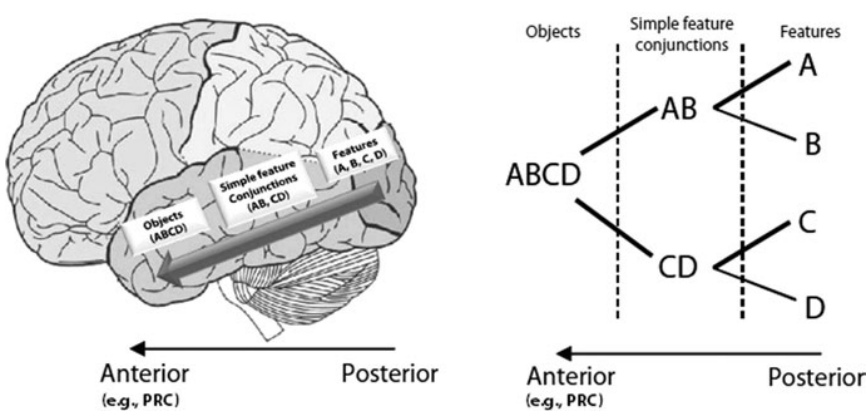

FIGURE 1. The representational-hierarchical theory. According to this view, the PRC sits at the apex of the ventral visual stream processing pathway, and contains complex representations of objects (Cowell et al., 2010a). The figure shows a lateral view of the human ventral visual stream, and the proposed organization of visual object representations within this pathway. A, B, C, and D represent distinct visual features. Simpler features are represented in posterior regions, and complex conjunctions of features (such as those comprising an entire object) are represented in anterior regions, including PRC. (Figure modified from Murray and Bussey, 1999 and Barense et al., 2012).

to the representational-hierarchical account, the PRC is the apex of this representational hierarchy, representing the most complex conjunctions-perhaps at the level of the whole object (Bussey and Saksida, 2002; Bussey and Saksida, 2007; Murray et al., 2007; Cowell et al., 2010a). In this view, the PRC participates in both perception and memory, and impairments following PRC damage can best be understood in terms of damage compromising high-level conjunctive representations (e.g., those comprising an object), leaving only lower-level representations (e.g., an object's shape or pattern) intact (Cowell et al., 2006; Lee et al., 2012). These impoverished representations will lead to impairments on both mnemonic and perceptual tasks.

Why is it advantageous to maintain representations of complex feature conjunctions? A stream of visual input (such as that encountered over a delay during a memory task) can create interference at the level of individual features, simply because different objects tend to share lower-level features (e.g., shapes, colors). However, the "conjunctive" PRC representations can resolve this interference at the feature level because they are unique to each individual object. Notably, Warrington and Weiskrantz proposed over 40 yrs ago that amnesia may be related to an increased vulnerability to interference (Warrington and Weiskrantz, 1970). Although this theory was later rejected (Warrington and Weiskrantz, 1978), there has been a recent resurgence in the idea (Cowan et al., 2004; Loewenstein et al., 2004; Wixted, 2004; Della Sala et al., 2005; Dewar et al., 2009; Bartko et al., 2010; McTighe et al., 2010). For example, rats with PRC lesions were impaired on a minimal-delay object recognition task when a "perceptually similar" object was introduced either before or after the to-be-remembered object. The rats were not impaired if the interfering item was "perceptually dissimilar" (Bartko et al., 2010). This suggests that in the absence of the complex object representations contained in PRC, the animals were unable to resolve interference from incidental, irrelevant lower-level object features. In the perceptually similar condition, many lower-level features were shared between the to-be-remembered object and the interfering object. The PRC bound these features into unique objects, and thus, intact animals were able to perform the task. However, when the PRC was lesioned, the intact posterior regions were not sufficient to resolve the perceptual similarity between low-level features. Similarly, the standard object recognition memory impairment observed after PRC lesions was rescued in rats by using a visual restriction procedure that reduced interference (McTighe et al., 2010). This finding was replicated in aged monkeys (Burke et al., 2011), aged rats (Burke et al., 2010, 2011) and in a mouse model of Alzheimer's disease (Romberg et al., 2012).

These findings were recently extended to humans with focal damage to the MTL using a novel perceptual discrimination task designed to tax complex object representations, for which fMRI implicated the PRC (Barense et al., 2012). Whereas amnesic cases whose damage was limited to the hippocampus performed normally, patients with MTL damage that included the PRC were vulnerable to object-based perceptual interference. These cases with PRC damage were impaired at successive complex object discriminations that contained many repeating low-level features, but critically, when we reduced the degree of object-level interference by interspersing perceptually dissimilar objects, we recovered their performance to normal levels (Barense et al., 2012). Healthy controls, who presumably had an intact PRC, performed as well under conditions of High Interference as they did under conditions of Low Interference; it was only the PRC-damaged group who were susceptible to the high levels of visual interference. These findings provide evidence to support the idea that the PRC is critical for representing the complex conjunctions of features that distinguish perceptually similar objects (see also Baxter, 2012; Peterson et al., 2012; Ryan et al., 2012). These PRC representations become essential when repeated presentation of multiple, similar features causes interference at the level of the features represented by intact posterior regions in the ventral visual stream.

In this study, we sought to determine whether these findings would generalize to another group of individuals with documented memory problems - those with MCI. The MTL is one of the earliest structures to show the neuropathological hallmarks of Alzheimer's disease, with the PRC demonstrating significant atrophy (Juottonen et al., 1998; Du et al., 2001; Guillozet et al., 2003; Pennanen et al., 2004; Taylor and Probst, 2008; Loewenstein et al., 2009; Schmidt-Wilcke et al., 2009). MCI patients who later convert to Alzheimer's disease show damage to the MTL (Bell-McGinty et al., 2005), including to the PRC (Mitchell et al., 2002). Interestingly, evidence suggests that MCI and Alzheimer's disease patients show impairments on perceptual tasks, especially when those tasks require complex visuoperceptual processing of objects (e.g., Alegret et al., 2009, 2010). Alegret et al. found that MCI and Alzheimer's disease patients have visuoperceptual deficits that may be more subtle than standard visuoperceptual tests can pick up, yet no study 
has investigated their performance on perceptual tasks known to be PRC dependent. To address this, here we administered the same perceptual discrimination task from Barense et al. (2012) to MCI patients. To provide further evidence in a related participant group and to increase our sample size, we also administered the task to older adults at risk for MCI, on the assumption that the integrity of the PRC would already be compromised in the earliest stages of the disorder (e.g., Du et al., 2001; Loewenstein et al., 2009; Schmidt-Wilcke et al., 2009). We identified these individuals at risk for MCI based on the Montreal Cognitive Assessment (MoCA), a brief standardized neuropsychological measure shown to be extremely sensitive in distinguishing controls from MCI patients (Nasreddine et al., 2005; Damian et al., 2011). Consistent with the cut-off scores provided by the developers of the MoCA (Nasreddine et al., 2005), we included older adults who scored below 26/30 on the MoCA as at risk for MCI, and older adults who scored 26/ 30 or above as a healthy control group. We predicted that MCI patients and individuals at risk for MCI would be impaired at object discrimination under conditions of high perceptual interference, but that we could improve their performance by reducing the degree of object-based perceptual interference.

\section{MATERIALS AND METHODS}

\section{Participants}

Ten patients with clinically diagnosed amnestic MCI participated in this study. These participants were recruited through the Emory Alzheimer's Disease Research Center, Atlanta, GA. Of these 10, three were excluded on the basis of their near perfect scores on the MoCA (Nasreddine et al., 2005) and MiniMental State Exam (MMSE, Folstein et al., 1975), leading us to believe they were "worried well" (Ahmed et al., 2008). Our exclusion criteria were a perfect score on one or both of the MoCA or the MMSE, as a well as a passing score on the remaining test (i.e., a perfect score on the MoCA and a passing score on the MMSE, or a perfect score on the MMSE and a passing score on the MoCA). The remaining seven patients (mean age $=68.43 \mathrm{yrs}$, standard deviation $=8.69$, three females) completed a detailed neuropsychological battery (Table 1). Patients were tested in the Memory and Aging laboratory at the Georgia Institute of Technology. All patients provided informed consent, and they were compensated for their time and travel expenses. The patient testing was approved by the Georgia Institute of Technology and the Emory University Institutional Review Boards.

In addition to MCI patients, 29 older adults with no known neurological conditions were recruited from the University of Toronto's Adult Volunteer Panel to participate in the study. Of these, 10 individuals (mean age $=71.11$, standard deviation $=$ 6.22, seven females) scored below 26 on the MoCA (mean score $=24.3$, standard deviation $=1.05)$ and were considered to be at risk for MCI (Nasreddine et al., 2005). The remaining 19 individuals (mean age $=71.05$ yrs, standard deviation $=$
5.33, 14 females) scored above 26 on the MoCA (mean score $=27.78$, standard deviation $=1.47$ ) and were included as control participants. Participants gave informed consent, and they were compensated for their time. These participants were tested in the Barense laboratory at the University of Toronto. The study was approved by the University of Toronto Ethics Review Board.

Thus, in total, three groups of participants participated in this study: those with MCI, older adults at risk for MCI ("At-risk" group), and older adults not thought to be at risk for MCI (healthy controls). Healthy controls' MoCA scores differed from those of the At-risk group $[t(27)=6.61, p<$ $0.001]$, and from those of MCI patients $[t(14)=4.21, p<$ 0.001]. MCI patients' performance on the MoCA was lower than the At-risk group $[t(15)=2.08, p=0.05]$. Because of scheduling constraints, two of the MCI patients were not administered the MoCA. Importantly, the MoCA was given after the experimental task for all remaining subjects, so the experimenter could not be biased by a participant's MoCA performance on the administration of the task. Age did not differ between controls, MCI patients, or those at risk for MCI $(t<0.21, p>0.49)$.

\section{Interference Task}

Participants performed a visual discrimination task described previously as Experiment 4 by Barense et al. (2012). Importantly, assessment of eye movements demonstrated that this task required a high degree of conjunctive processing (Experiment 1, Barense et al., 2012) and fMRI indicated that it recruited the PRC but not the hippocampus (Experiment 2, Barense et al., 2012). On each trial, participants determined if two stimuli were identical (a match) or different (a nonmatch). Each stimulus was presented in one of two invisible boxes $\left(\sim 9^{\circ} \times 9^{\circ}\right)$ positioned in the center of the screen, separated by $0.30^{\circ}$. The task was presented using E-Prime software (Psychology Software Tools, Pittsburgh, PA), and participants made their responses by using a button box connected through a USB port. The buttons were labeled such that participants could always see which button corresponded to which answer. Trials were self-paced, and stimuli were presented on screen until a response was made, for a maximum of $15 \mathrm{~s}$ for each trial. All participants completed a practice block with feedback after every trial until they felt comfortable with moving on to the experimental task.

The task was administered under two conditions (High Interference and Low Interference), with a short break between conditions. The key trials in both conditions were high ambiguity object discriminations involving abstract blob-like objects (similar to the blobs in Barense et al., 2005, 2012). These objects consisted of three distinct features: an inner shape, outer shape, and a fill pattern (Figs. 2a-c). Although low-level features (shapes, colors, and fill patterns) repeated across trials, all objects were trial-unique. On each trial, two objects were presented simultaneously, and all objects were rotated between $15^{\circ}$ and $165^{\circ}$ to discourage a simple feature matching strategy. On match trials, all three features were identical (e.g., $A B C$ vs. 
TABLE 1 .

Neuropsychological Test Results, Z-Scores in Parentheses

\begin{tabular}{|c|c|c|c|c|c|c|c|}
\hline Age (yrs) & 65 & 68 & 61 & 78 & 55 & 76 & 76 \\
\hline Gender & $\mathrm{F}$ & M & $\mathrm{F}$ & M & $\mathrm{M}$ & $\mathrm{F}$ & M \\
\hline Education (yrs) & 14 & 15 & 12 & 16 & 18 & 18 & 16 \\
\hline Diagnosis subtype & Amn Lang & Amn & Amn Lang & Amn Exec fx & Amn Lang Exec fx & Amn Lang & Amn \\
\hline MMSE & 24 & 23 & 28 & 28 & 30 & 30 & 29 \\
\hline GDS & 1 & 7 & 2 & 2 & 3 & - & - \\
\hline Beck Depression Inventory & 4 & - & 1 & - & 12 & - & - \\
\hline JOLO & 16 & - & 20 & 22 & 24 & - & 22 \\
\hline Logical memory I & $15(-0.16)$ & $5(-2.70)$ & $14(0.26)$ & $11(-0.78)$ & $9(-1.96)$ & $14(-0.33)$ & $8(-1.57)$ \\
\hline Logical memory II & $13(-0.33)$ & $4(-2.39)$ & $12(0.12)$ & $10(-0.67)$ & $8(-1.82)$ & $13(-0.23)$ & $6(-1.64)$ \\
\hline BVMT-R total recall & $16(-1.10)$ & - & $25(0.40)$ & $15(0.90)$ & $13(-2.00)$ & - & $18(-0.40)$ \\
\hline Trails B & $117(0)$ & $171(-1.80)$ & $162(-1.90)$ & $102(-0.10)$ & $43(1.10)$ & $53(1.3)$ & $95(-0.10)$ \\
\hline Verbal fluency: FAS & $23(-1.90)$ & - & $30(-0.80)$ & $24(-1.50)$ & $47(0.10)$ & - & $30(-0.80)$ \\
\hline Category fluency: animals & $19(-0.30)$ & $17(-0.20)$ & $16(-0.80)$ & $10(-2.30)$ & $34(2.30)$ & $18(-0.5)$ & $10(-2.30)$ \\
\hline WAIS-R digit symbol & $45(0.00)$ & $36(0.00)$ & $66(1.33)$ & $37(0.00)$ & $64(1.00)$ & $59(2.00)$ & $41(0.33)$ \\
\hline Boston naming test (no cue) & $25(-1.60)$ & $30(2.40)$ & $21(-1.80)$ & $25(-0.90)$ & $25(-1.80)$ & $18(-2.40)$ & - \\
\hline Digit span: forward & $6(-0.92)$ & $10(1.41)$ & $7(-0.39)$ & $9(-0.33)$ & $11(1.55)$ & $11(2.33)$ & - \\
\hline Digit span: backward & $5(-0.50)$ & $6(0.25)$ & $4(-0.99)$ & $3(-0.33)$ & $10(1.65)$ & $9(2.33)$ & - \\
\hline Clock drawing & $11(0.52)$ & $12(1.63)$ & $13(2.74)$ & $8(-0.69)$ & $13(3.48)$ & $13(1.54)$ & - \\
\hline
\end{tabular}

"Amn" means single-domain amnestic, "Lang" means amnestic plus language, "Exec fx" means amnestic plus executive function. Individual cells for each patient represent raw data scores, parentheses reflect Z-scores. "-" indicates no data is available for that patient on that test. Neuropsychological tests: BVMT-R, Benton Verbal Memory Test—Revised (Benton et al., 1983); CDR, Clinical Dementia Rating (Morris, 1993); CVLT, California Verbal Learning Test (Delis et al., 2000); GDS, Global Deterioration Scale (Reisberg et al., 1982); the Beck Depression Inventory (Beck et al., 1961); JOLO, Judgment of Line Orientation (Benton et al., 1978); Logical Memory I and II (Wechsler, 1945); MMSE, Mini Mental Status Exam (Folstein et al., 1975); MoCA, Montreal Cognitive Assessment (Nasreddine et al., 2005); WAIS-R, Wechsler Adult Intelligent Scales—Revised (Wechsler, 1981), Boston Naming Test (Kaplan et al., 1983); Digits Forward; Digits Backward; and a Clock Drawing Test.

ABC; letters represent individual features). Critically, for nonmatch trials, only one feature differed (e.g., $\mathrm{ABC}$ vs. ABD; whether inner shape, outer shape, or fill pattern differed was fully counterbalanced). This high degree of feature ambiguity placed demand on high-level conjunctive representations and analysis of the object as a whole (Barense et al., 2012). The High Interference condition contained 88 consecutive trials of high ambiguity object discriminations (44 match and 44 nonmatch trials, intermixed in a pseudorandom sequence; Fig. $2 \mathrm{~b}$ ). Each Low Interference block also contained 88 trials, but unlike the High Interference condition, these blocks contained 30 high ambiguity object trials (15 match, 15 non-match) that were each interspersed with two trials containing photographs of easily discriminable everyday objects (58 trials, 29 match, 29 nonmatch; Figs. 2a,c). Importantly, the stimuli presented on photograph trials shared minimal features with the blob-like objects (i.e., they were color photographs of everyday objects, not black and white line-drawings) and thus, the degree of accumulated perceptual interference was much lower in the Low Interference condition. As such, we predicted that the
MCI patient group and the At-risk group would perform better on the Low Interference condition relative to the High Interference condition. We administered two blocks of the Low Interference condition, one before and one after the High Interference condition, to ensure that any observed deficits were specific to the buildup of interfering features and not fatigue or generic task-practice effects.

\section{Control Task: Difficult Size}

Following the completion of the interference task, participants were administered a control task that did not require conjunctive processing, but was matched in terms of difficulty to the High Interference condition (control task used previously in Barense et al., 2012). The procedures for the control condition were identical to the interference task described above except the stimuli were squares and participants indicated if they were identical (match) or different (nonmatch) (Fig. 2d). For nonmatch trials, the length of each side of the square was randomly varied from 67 to 247 pixels. The difference between 


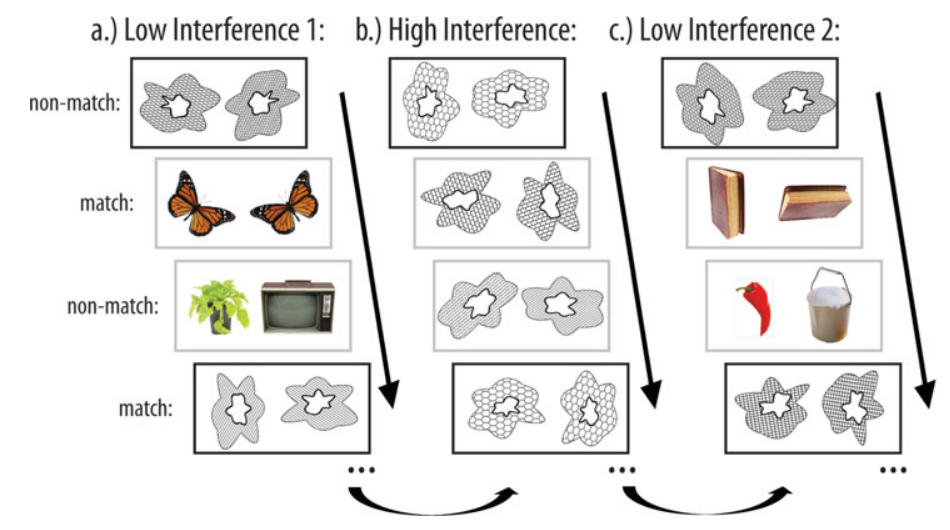

d.) Difficult Size Control Task:

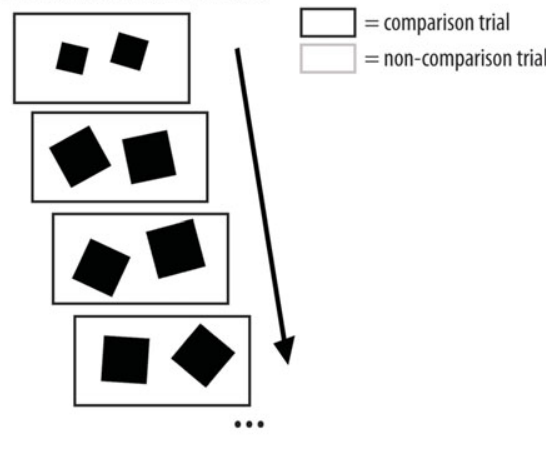

FIGURE 2. Participants indicated whether two simultaneously presented stimuli were a match or a nonmatch. All objects were rotated, and participants decided whether the objects were identical (a match) or different (a nonmatch). The critical trials were high ambiguity object discriminations involving blob-like objects. These objects were defined by three features: inner shape, outer shape, and fill pattern. For high ambiguity nonmatch trials, only one of these three features differed, and thus, these trials placed a demand on high-level conjunctive representations and analysis of the object as a whole. (a, c) For the Low Interference condition, a high ambiguity object trial was always followed by two trials involving perceptually distinct, colored objects (30 high ambiguity object trials in total).

the lengths of the squares varied between 9 and 15 pixels. For match trials, the two rotated squares were identical in size.

\section{Planned Analysis: Interference Task}

To examine performance on the interference task, we calculated a discriminability measure $\left(d^{\prime}\right)$, where correct responses of "different" to images that were different were designated as hits, and incorrect responses of "different" to images that were the same were designated as false alarms (MacMillan and Creelman, 1991). Scores of $100 \%$ or $0 \%$ for Hits and False Alarms were subjected to a standard correction whereby half a trial was either subtracted or added, respectively, to the actual number of trials that were designated Hits or False Alarms. Importantly, when calculating $d$, we analyzed only the high ambiguity discrimination trials and did not include the photo object trials (for which performance was at ceiling). Thus, our critical trials were 88 trials in the High Interference condition and 30 trials in each Low Interference condition. These data were subjected to a $3 \times 3$ repeated measures ANOVA with a within-subject factor of condition (Low Interference 1, High Interference, Low Interference 2) and a between-subject factor of group (MCI patients, At-risk group, healthy controls). We predicted that MCI patients and the At-risk group would be impaired on the High Interference, but not Low Interference, conditions, compared with controls. Given our directional hypotheses, all statistical tests on the experimental data were one-tailed unless stated otherwise.

\section{Planned Analyses: Control Tasks}

We calculated $d^{\prime}$ for the difficult size task in the same manner as described above. We then performed independent $t$-tests (b) The High Interference condition was a straight block of 88 consecutive high ambiguity object trials. To avoid confounding effects of fatigue, the order of testing conditions was Low Interference 1, High Interference, and Low Interference 2. We compared performance on high ambiguity trials only. All objects were trial-unique, though the individual features (e.g., shape segments, fill patterns) repeated across trials. (d) In the Difficult Size control task, participants decided if two rotated squares were the same size. This condition could be solved on the basis of a single feature and did not tax conjunctive object representations. Healthy control participants found this condition to be more difficult than the High Interference condition. (Figure modified from Barense et al., 2012.)

to compare performance of MCI patients and those at risk for MCI to healthy controls. We predicted no significant differences in performance across groups.

\section{RESULTS}

\section{Interference Task}

Data from the interference task are shown in Figure 3. The results of our $3 \times 3$ repeated measures ANOVA (interference condition $\times$ group) revealed main effects of interference $[F(2$, $66)=15.79, p<0.001]$ and group $[F(2,33)=3.07, p=$ $0.03]$, and an interference $\times$ group interaction $[F(4,66)=$ 1.99, $p=0.05$ ]. Follow up $t$-tests showed that, as predicted, both the MCI patients and the At-risk group were impaired on the High Interference condition, compared with healthy controls $[t>2.20, p<0.02]$, with no differences between the MCI patients and the At-risk group $(t(15)=0.73, p=0.48$, two-tailed). In contrast, by reducing the degree of perceptual interference, we were largely able to improve performance in both groups: neither group was impaired on the first Low Interference condition $[t<0.81, p>0.21]$ nor were the MCI patients impaired on the second Low Interference condition $[t(24)=0.80, p=0.22]$. Unexpectedly, the At-risk group was impaired on the second Low Interference condition $[t(27)=$ 1.99, $p=0.03$ ]. Because the analyzed trials from the Low Interference condition still contained objects with low-level features (e.g., shape segments, fill patterns) that had repeated from previous conditions, a potential explanation for this impairment is that these At-risk participants were unable to recover from 


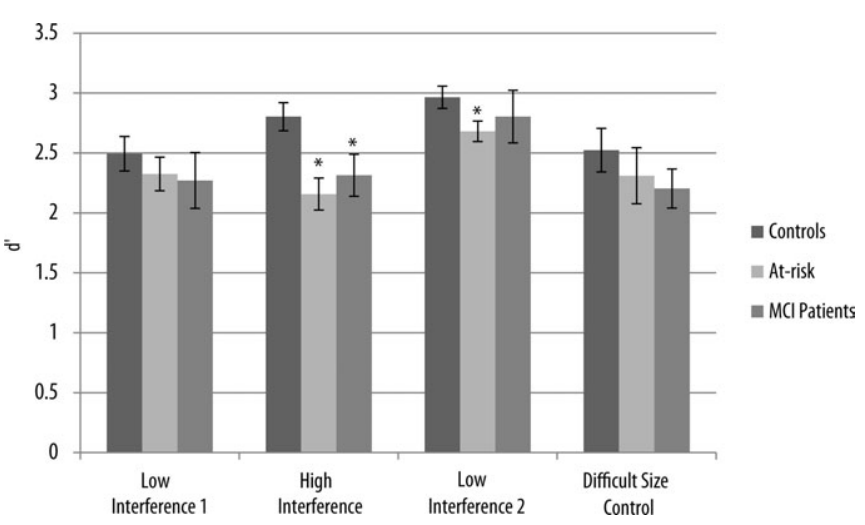

FIGURE 3. $d$ ' scores for each group. MCI patients and the Atrisk group were impaired on the High Interference condition, but their performance was largely rescued by reducing the degree of interference. There were no differences in performance on the Difficult Size control task, suggesting that the observed deficits are not driven by task difficulty. Error bars denote standard error of the mean. ${ }^{*} P<\mathbf{0 . 0 5}$ (comparisons of the MCI patient group and Atrisk group relative to healthy controls).

the interference experienced in the previous High Interference condition. Future work is needed to establish the time course of these interference effects.

Reaction time data and proportion correct split according to match and nonmatch trials are shown in Table 2.

\section{Control Task: Difficult Size}

We compared performance of the At-risk group and MCI patients to healthy controls using independent $t$-tests and found that neither group was impaired [ $t<0.94, p>0.18]$ (Fig. 3). A paired $t$-test showed no evidence that control participants found the Difficult Size control as difficult as High Interference condition $[t(18)=1.40, p=0.18$, two-tailed]. Thus, the intact performance of the memory-impaired groups on the Difficult Size discriminations indicates that their impairment on the High Interference condition was not simply due to fatigue from performing consecutive difficult discriminations. Table 2 shows the reaction time and proportion correct for match and nonmatch trials.

\section{DISCUSSION}

In this study, we tested amnestic MCI patients, older adults at risk for $\mathrm{MCI}$, and healthy older adult controls on an object discrimination task that has been shown to recruit the PRC and to emphasize processing conjunctions of features (Barense et al., 2012). We administered the task under conditions of high perceptual interference (many repeating similar visual features shown across trials) and low perceptual interference (visually dissimilar features interspersed across trials). MCI patients and those at risk for MCI were impaired under conditions of High Interference, but critically, when we minimized perceptual interference by reducing the number of similar features viewed across trials, their performance improved. Notably, the deficits could not be attributed to generic task difficulty, as both MCI patients and At-risk participants performed normally on a single-feature discrimination task that was matched in difficulty to the High Interference condition. This study reveals striking convergence with findings in amnesic patients whose focal MTL lesions included the PRC (Barense et al., 2012) (see Supporting Information Fig. 1 for a direct comparison), and suggests that memory disorders involving the MTL may reflect a heightened vulnerability to visual interference.

These findings, when considered together with research in humans with focal MTL damage (Barense et al., 2012) and animals with selective PRC lesions (McTighe et al., 2010), provide support for the view that the PRC represents the conjunctions of features necessary to distinguish perceptually similar objects. In the Low Interference conditions, the MCI patients may have successfully used a single-feature strategy supported

TABLE 2.

Reaction Time and Accuracy (Proportion Correct) for Each Participant Group

\begin{tabular}{|c|c|c|c|c|}
\hline & $\begin{array}{c}\text { Low } \\
\text { Interference } 1\end{array}$ & $\begin{array}{c}\text { High } \\
\text { Interference }\end{array}$ & $\begin{array}{c}\text { Low } \\
\text { Interference } 2\end{array}$ & $\begin{array}{l}\text { Difficult Size } \\
\text { Control Task }\end{array}$ \\
\hline \multicolumn{5}{|c|}{ Accuracy: match trials } \\
\hline Controls & $0.82(0.13)$ & $0.83(0.11)$ & $0.90(0.07)$ & $0.81(0.17)$ \\
\hline At-risk & $0.79(0.15)$ & $0.73(0.17)$ & $0.90(0.07)$ & $0.79(0.16)$ \\
\hline MCI patients & $0.81(0.10)$ & $0.78(0.14)$ & $0.90(0.08)$ & $0.78(0.09)$ \\
\hline \multicolumn{5}{|c|}{ Accuracy: nonmatch trials } \\
\hline Controls & $0.72(0.21)$ & $0.83(0.11)$ & $0.79(0.12)$ & $0.76(0.13)$ \\
\hline At-risk & $0.71(0.16)$ & $0.75(0.10)$ & $0.71(0.09)$ & $0.71(0.12)$ \\
\hline MCI patients & $0.69(0.14)$ & $0.74(0.13)$ & $0.71(0.07)$ & $0.75(0.05)$ \\
\hline \multicolumn{5}{|c|}{ Reaction times: all trials } \\
\hline Controls & $5,925.27(2,002.27)$ & $5,995.20(1,277.71)$ & 6,130.72 (1,788.44) & $3,017.81(1,091.72)$ \\
\hline At-risk & $6,639.39(1,550.92)$ & $6,695.56(1,347.78)$ & $6,724.54(1,370.30)$ & $2,858.29(967.05)$ \\
\hline MCI patients & $7,551.99(1,656.85)$ & 7,396.72 (999.79) & $7,089.37(1,493.05)$ & $2,970.14(562.85)$ \\
\hline
\end{tabular}

Standard deviations shown in parentheses. 
by intact regions upstream in the ventral visual stream (by definition, objects in the discrimination of $\mathrm{ABC}$ vs. $\mathrm{ABD}$ differed by a single feature: $C$ vs. D, see Fig. 1). However, the repeated administration of multiple low-level object features (e.g., shapes, fill patterns) — as was the case in the High Interference condition-created massive interference at the level of the representations for these individual features. Under these conditions, a more complex representation that binds the features into unique objects is necessary to resolve the interference from repeating features. Put differently, when a constant stream of visual information creates large-scale feature-level interference from irrelevant features processed on previous trials, a more elaborate object-level representation will be resistant to the feature-level interference. We propose this more complex objectlevel representation to be contained within PRC. In addition to processing the complex conjunction of features comprising the object, recent research suggests that the PRC may also protect from visual interference by "suppressing" the representation of the lower-level features represented in earlier regions of the visual stream (Peterson et al., 2012).

These results complement a growing body of evidence suggesting that memory impairments following MTL damage may be due to an increased vulnerability to interference (Cowan et al., 2004; Loewenstein et al., 2004; Della Sala et al., 2005; Dewar et al., 2009). For example, one study found that recall of a story in patients with MCI was increased by a remarkable $35 \%$ under conditions of Low Interference (during the delay participants reclined in a dark, quiet room) compared with recall under standard interference (a 1-h delay filled with psychometric tasks) (Della Sala et al., 2005). A similar result was reported for amnesics with focal brain damage (Cowan et al., 2004). The interference in these studies may have been from interfering mental activity and memory formation (Wixted, 2004), and/or it may have been due to interference from similar visual information processed during the psychometric tasks. Consistent with the latter idea of visual interference from perceptually similar information, sensory restriction rescued an object recognition memory deficit in rats with perirhinal lesions (McTighe et al., 2010), aged rats (Burke et al., 2010), and in a mouse model of Alzheimer's disease-typical amyloid- $\beta$ pathology (the tgCRND8 mouse, Romberg et al., 2012). The present results extend these findings from the mnemonic domain to the visual discrimination of trial-unique stimuli.

Although we prefer to consider these results in terms of impoverished object representations impairing perception, it is worth noting that there is a working memory component to the task. Although the objects were displayed simultaneously, the discriminations required a series of eye movements and comparisons across objects. Thus, it is possible that the observed deficit could be related to problems with working memory. There has been a recent surge of research indicating that short-term memory may be impaired following MTL damage (e.g., Hannula et al., 2006; Nichols et al., 2006; Olson et al., 2006; Warren et al., 2010; although see Jeneson et al., 2010), with evidence suggesting that the MTL is important in online processing of highly similar visual objects (Warren et al., 2011a,b). We are certainly not opposed to the notion that MTL structures are important for working memory. Indeed, it seems highly likely that the conjunctive representations processed by PRC are essential for online maintenance of information while shifting attention from one object to the other (see also Barense et al., 2012). We would additionally argue, however, that these representations are critical for any cognitive task that requires them, which includes long-term memory, working memory, and complex object perception. In support of this, other studies have demonstrated perceptual deficits on tasks with no working memory component (i.e., perception of single objects and figure-ground perception of familiar object configuration), suggesting that the deficits observed here reflect a more fundamental deficit in representing complex objects (Lee and Rudebeck, 2010; Barense et al., 2011b).

It is important to note that due to the unavailability of structural MRI scans of participants in the current study, we cannot conclusively localize the observed deficits to PRC pathology. It is very well established that the hippocampus and entorhinal cortex are affected very early, likely even first, in MCI (Wakabayashi et al., 1994; Harris et al., 2010; Devanand et al., 2012). The PRC is heavily connected to these structures (e.g., Suzuki and Amaral, 1994), and is part of the neural circuit affected early in MCI (Mitchell et al., 2002; Bell-McGinty et al., 2005; Schuff and Zhu, 2007; Zhang et al., 2012). Thus, it is not surprising that PRC function would be compromised. Consistent with this, the profile of performance in both the MCI patients and those at risk for MCI is remarkably consistent with findings from research that allows more precise localization of the PRC: (1) animal studies that have demonstrated object discrimination deficits and interference effects after precise, localized PRC lesions (Buckley et al., 2001; Bussey et al., 2002, 2003; Bartko et al., 2010; McTighe et al., 2010), and (2) functional neuroimaging revealing PRC activity in healthy participants during the discrimination task reported here (Barense et al., 2012) and other tasks taxing complex object perception (Devlin and Price, 2007; Lee et al., 2008; O'Neil et al., 2009; Barense et al., 2010a, 2011a). In addition, based on the intact performance of amnesic patients with focal hippocampal damage (Barense et al., 2012; Supporting Information Fig. 1), we can localize our deficits to nonhippocampal structures.

One notable finding of the present study is that although the At-risk participants do not have diagnosed MCI, their performance suggests that a clinical exam might lead to an MCI diagnosis. However, now, they appear to be "unworried unwell." In other words, these volunteers from the community presented with no subjective memory complaint, but had an objective cognitive deficit. The subjective memory complaint in question is a vital component of diagnosis using the original Peterson criteria (Petersen et al., 1999), and it remains to be seen whether these participants would be diagnosed with MCI. Nonetheless, the MoCA has been identified as a sensitive brief measure in detecting cognitive impairment (Nasreddine et al., 2005; Markwick et al., 2012). Additionally, the present study indicates that and those who fall below norms on the MoCA are vulnerable to visual interference. In fact, an additional 
correlational analysis investigating performance across all groups on all tasks showed that MoCA scores predicted performance on the High Interference task $(r(33)=0.54, p<0.01$, Bonferroni corrected), but not the other three conditions $[r(33)$ $<0.29, p>0.09$, Bonferroni corrected]. We are currently following up the At-risk participants on a number of cognitive and physiological longitudinal assessments.

In conclusion, these data illustrate that reducing perceptual interference improves performance on a discrimination task in MCI patients and those at risk for MCI. Furthermore, these data add additional support to the idea that damage to the complex, conjunctive representations processed by PRC causes an increased susceptibility to object-based perceptual interference, which leads to deficits in both memory and perception.

\section{Acknowledgments}

The authors thank all participants for their time, and Sinead Brady and Alina Guna (University of Toronto) for help with data collection.

\section{REFERENCES}

Ahmed S, Mitchell J, Arnold R, Dawson K, Nestor P, Hodges J. 2008. Memory complaints in mild cognitive impairment, worried well, and semantic dementia patients. Alzheimer Dis Assoc Disord 22:227-235.

Alegret M, Boada-Roviera M, Vinyes-Junque G, Valero S, Espinosa A, Hernandez I, Modinos G, Rosende-Roca M, Mauleon A, Becker J, Tarraga L. 2009. Detection of visuoperceptual deficits in preclinical and mild Alzheimer's Dsisease. J Clin Exp Neuropsychol 31:860-867.

Alegret M, Vinyes-Junqué G, Boada M, Martinez-Lage P, Cuberas G, Espinosa A, Roca I, Hernández I, Valero S, Rosende-Roca M, Mauleón A, Becker JT, Tárraga L. 2010. Brain perfusion correlates of visuoperceptual deficits in mild cognitive impairment and mild Alzheimer's disease. J Alzheimer Dis 21:557-567.

Barense M, Bussey T, Lee A, Rogers T, Davies R, Saksida L, Murray E, Graham K. 2005. Functional specialization in the human medial temporal lobe. J Neurosci 25:10239-10246.

Barense M, Gaffan D, Graham K. 2007. The human medial temporal lobe processes online representations of complex objects. Neuropsychologia 45:2963-2974.

Barense M, Henson R, Lee A, Graham K. 2010a. Medial temporal lobe activity during complex visual discrimination of faces, objects and scenes: The effect of viewpoint. Hippocampus 20:389-401.

Barense M, Rogers T, Bussey T, Saksida L, Graham K. 2010b. Influence of conceptual knowledge on visual object discrimination: Semantic dementia and MTL amnesia. Cereb Cortex 20:2568-2582.

Barense M, Henson R, Graham K. 2011a. Perception and conception: Temporal lobe activity during complex discriminations of familiar and novel faces and objects. J Cogn Neurosci 23:3052-3057.

Barense M, Ngo J, Hung L, Peterson M. 2011b. Interactions of memory and perception in amnesia: The figure-ground perspective. Cereb Cortex 22:11.

Barense M, Groen I, Lee A, Yeung L, Brady S, Gregori M, Kapur N, Bussey T, Saksida L, Henson R. 2012. Intact memory for irrelevant information impairs perception in amnesia. Neuron 75:157-167.

Bartko S, Winters B, Cowell R, Saksida L, Bussey T. 2007a. Perceptual functions in perirhinal cortex in rats: Zero-delay object recognition and simultaneous oddity discriminations. J Neurosci 27: 2548-2559.
Bartko S, Winters B, Cowell R, Saksida L, Bussey T. 2007b. Perirhinal cortex resolves feature ambiguity in configural object recognition and perceptual oddity tasks. Learn Mem 14:821-832.

Bartko S, Cowell R, Winters B, Bussey T, Saksida L. 2010. Heightened susceptibility to interference in an animal model of amnesia: Impairment in encoding, storage, retrieval —or all three? Neuropsychologia 48:2987-2997.

Baxter M. 2012. It's all coming back to me now: Perception and memory in amnesia. Neuron 75:8-10.

Bell-McGinty S, Lopez O, Meltzer C, Scanlon J, Whyte E, DeKosky S, Becker J. 2005. Differential cortical atrophy in subgroups of Mild Cognitive Impairment. Arch Neurol 62:1393-1397.

Buckley M, Booth M, Rolls E, Gaffan D. 2001. Selective perceptual impairments after perirhinal cortex ablation. J Neurosci 21: 9824-9836.

Burke S, Wallace J, Nematollahi S, Uprety A, Barnes C. 2010. Pattern separation deficits may contribute to age associated recognition impairments. Behav Neurosci 124:559-573.

Burke S, Wallace J, Hartzell A, Nematollahi S, Plange K, Barnes C. 2011. Age-associated deficits in pattern separation functions of the perirhinal cortex: A cross-species consensus. Behav Neurosci 125:836-47.

Bussey T, Saksida L. 2002. The organization of visual object representations: A connectionist model of effects of lesions in perirhinal cortex. Eur J Neurosci 15:355-364.

Bussey T, Saksida L. 2007. Memory, perception, and the ventral visual perirhinal hippocampal stream: Thinking outside of the boxes. Hippocampus 908:898-908.

Bussey T, Saksida L, Murray E. 2002. Perirhinal cortex resolves feature ambiguity in complex visual discriminations. Eur J Neurosci 15:363-374.

Bussey T, Saksida L, Murray E. 2003. Impairments in visual discrimination after perirhinal cortex lesions: Testing 'declarative' vs. 'perceptualmnemonic' views of perirhinal cortex function. Eur J Neurosci 17.

Clark R, Reinagel P, Broadbent N, Flister E, Squire L. 2011. Intact performance on feature-ambiguous discriminations in rats with lesions of the perirhinal cortex. Neuron 70:132-140.

Cowan N, Beschin N, Della Sala S. 2004. Verbal recall in amnesiacs under conditions of diminished retroactive interference. Brain 127:825-834.

Cowell R, Bussey T, Saksida L. 2006. Why does brain damage impair memory? A connectionist model of object recognition memory in perirhinal cortex. J Neurosci 26:12186-12197.

Cowell R, Bussey T, Saksida L. 2010a. Components of recognition memory: Dissociable cognitive processes or just difference in representational complexity? Hippocampus 20:1245-1262.

Cowell R, Bussey T, Saksida L. 2010b. Functional dissociations within the ventral object processing pathway: Cognitive modules or a hierarchical continuum? J Cogn Neurosci 22:2460-2479.

Cowell RA. 2012. Computational models of perirhinal cortical function. Hippocampus 22:1952-1964.

Damian A, Jacobsen S, Hentz J, Belden C, Shill H, Sabbagh M, Caviness J, Adler C. 2011. The Montreal Cognitive Assessment and the Mini-Mental State Examination as screening instruments for cognitive impairment: Item analyses and threshold scores. Dement Geriatr Cogn Disord 31:126-131.

Della Sala S, Cowan N, Beschin N, Perini M. 2005. Just lying there, remembering: Improving recall of prose in amnesic patients with mild cognitive impairment by minimising interference. Memory $13: 435-440$

Desimone R, Ungerleider L. 1989. Neural mechanisms of visual processing in monkeys. In: Boller F, Grafman J, editors. Handbook of Neuropsychology. New York: Elsevier Sciences. pp 267-299.

Devanand D, Bansal R, Liu J, Hao X, Pradhaben G, Peterson B. 2012. MRI hippocampal and entorhinal cortex mapping in predicting conversion to Alzheimer's disease. Neuroimage 60: 1622-1629. 
Devlin J, Price C. 2007. Perirhinal contributions to human visual perception. Curr Biol 17:1484-1488.

Dewar M, Garcia Y, Cowan N, Della Sala S. 2009. Delaying interference enhances memory consolidation in amnesic patients. Neuropsychology 23:627-634.

Du A, Schuff N, Amend D, Laakso M, Hsu Y, Jagust W, Yaffe K, Kramer J, Reed B, Norman D, Chui HC, Weiner MW. 2001. Magnetic resonance imaging of the entorhinal cortex and hippocampus in mild cognitive impairment and Alzheimer's disease. J Neurol Neurosurg Psychiatry 71:441-447.

Eichenbaum H, Cohen N. 2001. From Conditioning to Conscious Recollection: Memory Systems of the Brain. New York: Oxford University Press.

Folstein M, Folstein S, McHugh P. 1975. "Mini-Mental State": A practical method for grading the cognitive state of patients for the clinician. J Psychiatr Res 12:189-198.

Graham K, Barense M, Lee A. 2010. Going beyond LTM in the MTL: A synthesis of neuropsychological and neuroimaging findings on the role of the medial temporal lobe in memory and perception. Neuropsychologia 48:831-853.

Guillozet A, Weintraub S, Mash D, Mesulam M. 2003. Neurofibrillary tangles, amyloid, and memory in aging and mild cognitive impairment. Arch Neurol 60:729-736.

Harris J, Devidze N, Verret L, Ho K, Halabisky B, Thwin M, Kim D, Hamto P, Lo I, Yu G, Palop J, Masliah E, Mucke L. 2010. Transsynaptic progression of amyloid-B-induced neuronal dysfunction within the entorhinal-hippocampal network. Neuron 68:428-41.

Juottonen K, Laakso M, Insausti R, Lehtovirta M, Pitkanen A, Partanen K, Soininen H. 1998. Volumes of entorhinal and perirhinal cortices in Alzheimer's disease. Neurobiol Aging 19:15-22.

Kim S, Jeneson A, van der Horst A, Frascino J, Hopkins R, Squire L. 2011. Memory, visual discrimination performance, and the human hippocampus. J Neurosci 31:2624-2630.

Lee A, Barense M, Graham K. 2005a. The contribution of the human medial temporal lobe to perception: Bridging the gap between animal and human studies. Q J Exp Psychol 58b:300-325.

Lee A, Bussey T, Murray E, Saksida L, Epstein R, Kapur N, Hodges J, Graham K. 2005b. Perceptual deficits in amnesia: Challenging the medial temporal lobe 'mnemonic' view. Neuropsychologia 43:1-11.

Lee A, Rudebeck S. 2010. Human medial temporal lobe damage can disrupt the perception of single objects. J Neurosci 30:6588-6594.

Lee A, Scahill V, Graham K. 2008. Activating the medial temporal lobe during oddity judgment for faces and scenes. Cereb Cortex 18:683-696.

Lee A, Yeung L-K, Barense M. 2012. The hippocampus and visual perception. Front Hum Neurosci 6:91.

Loewenstein D, Acevedo A, Luis C, Crum T, Barker W, Duara R. 2004. Semantic interference deficits and detection of mild Alzheimer's disease and mild cognitive impairment without dementia. J Int Neuropsychol Soc 10:91-100.

Loewenstein D, Acevedo A, Potter E, Schinka J, Raj A, Greig M, Agron J, Barker W, Wu Y, Small B, Schofield E, Duara R. 2009. Severity of medial temporal lobe atrophy and amnestic mild cognitive impairment: Selecting type and number of memory tests. American J Geriatr Psychiatry 17:1050-1058.

MacMillan N, Creelman C. 1991. Detection Theory: A User's Guide. New York: Cambridge University Press.

Markwick A, Zamboni G, de Jager C. 2012. Profiles of cognitive subtest impairment in Montreal Cognitive Assessment (MoCA) in a research cohort with normal Mini-Mental State Examination (MMSE) scores. J Clin Exp Neuropsychol 34:750-757.

Martinovic J, Gruuber T, Muller M. 2008. Coding of visual object features and feature conjunction in the human brain. PLoS ONE 3:e3781.

McTighe S, Cowell R, Winters B, Bussey T, Saksida L. 2010. Paradoxical false memory for objects after brain damage. Science 330:1408-1410.
Mitchell T, Mufson E, Schneider J, Cochran E, Nissanov J, Han L, Bienias J, Lee V, Trojanowski J, Bennett D, Arnold S. 2002. Parahippocampal tau pathology in healthy aging, mild cognitive impairment, and early Alzheimer's disease. Ann Neurol 51: 182-189.

Murray E, Bussey T. 1999. Perceptual-mnemonic functions of the perirhinal cortex. Trends Cogn Sci 3:142-151.

Murray E, Bussey T, Saksida L. 2007. Visual perception and memory: A new view of medial temporal lobe function in primates and rodents. Annu Rev Neurosci 30:99-122.

Murray E, Wise S. 2012. Why is there a special issue on perirhinal cortex in a journal called Hippocampus?: The perirhinal cortex in historical perspective. Hippocampus 22:1941-1951.

Nasreddine Z, Phillips N, Bedirian V, Charbonneau S, Whitehead V, Collin I, Cummings J, Chertkow H. 2005. The Montreal Cognitive Assessment, MoCA: A brief screening tool for mild cognitive impairment. J Am Geriatr Soc 53:695-699.

O’Neil E, Cate A, Kohler S. 2009. Perirhinal cortex contributes to accuracy in recognition memory and perceptual discriminations. J Neurosci 29:8329-8334.

O’Neil E, Protzner A, McCormick C, McLean D, Poppenk J, Cate A, Kohler S. 2011. Distinct patterns of functional and effective connectivity between perirhinal cortex and other cortical regions in recognition memory and perceptual discrimination. Cereb Cortex 22:74-85.

Pennanen C, Kivipelto M, Tuomainen S, Hartikainen P, Hanninen T, Laakso M, Hallikainen M, Vanhanen M, Nissinen A, Helkala E, Vainio P, Vanninen R, Partanen K, Soininen H. 2004. Hippocampus and entorhinal cortex in mild cognitive impairment and early AD. Neurobiol Aging 25: 303-310.

Peterson M, Cacciamani L, Barense M, Scalf P. 2012. The perirhinal cortex modulates V2 activity in response to agreement between part familiarity and configuration familiarity. Hippocampus 22:1965-1977.

Petersen R, Smith G, Waring S, Ivnik R, Tangalos E, Kokmen E. 1999. Mild cognitive impairment: Clinical characterization and outcome. Arch Neurol 56:303-308.

Riesenhuber M, Poggio T. 1999. Hierarchical models of object recognition in cortex. Nat Neurosci 2:1019-1025.

Romberg C, McTighe S, Heath C, Whitcomb D, Cho K, Bussey T, Saksida L. 2012. False recognition in a mouse model of Alzheimer's disease: Rescue with sensory restriction and memantine. Brain 135:2103-2114.

Ryan L, Cardoza J, Barense M, Kawa K, Walletin-Flores J, Alexander G, Barnes C. 2012. Age-related impairments in a complex object discrimination task that engages perirhinal cortex. Hippocampus 22:1978-1989.

Saksida L, Bussey T. 2010. The representational-hierarchical view of amnesia: Translation from animal to human. Neuropsychologia 48:2370-2384.

Schmidt-Wilcke T, Poljansky S, Hierlmeier S, Hausner J, Ibach B. 2009. Memory performance correlates with gray matter density in the ento-perirhinal cortex and posterior hippocampus in patients with mild cognitivei mpairment and healthy controls - a voxel based morphometry study. Neuroimage 47:1914-1920.

Schuff N, Zhu X. 2007. Imaging of mild cognitive impairment and early dementia. Br J Radiol 80:S109-S114.

Squire L, Wixted J. 2011. The cognitive neuroscience of human memory since HM. Annu Rev Neurosci 34:259-288.

Squire L, Zola-Morgan S. 1991. The medial temporal lobe memory system. Science 253:1380-1386.

Tanaka K. 1996. Inferotemporal cortex and object vision. Annu Rev Neurosci 19:109-139.

Taylor K, Probst A. 2008. Anatomic localization of the transentorhinal region of the perirhinal cortex. Neurobiol Aging 29:1591-1596.

Wakabayashi K, Honer W, Masliah E. 1994. Synapse alterations in the hippocampal-entorhinal formation in Alzheimer's disease with and without Lewy body disease. Brain Res 19:24-32. 
Warren D, Duff M, Jensen U, Tranel D, Cohen N. 2012. Hiding in plain view: Lesions of the medial temporal lobe impair online representation. Hippocampus 22:1577-1588.

Warren D, Duff M, Tranel D, Cohen N. 2011b. Observing degradation of visual representations over short intervals when medial temporal lobe is damaged. J Cogn Neurosci 23:3862-3873.

Warrington E, Weiskrantz L. 1970. Amnesic syndrome: Consolidation or retrieval? Nature 228:628-630.
Warrington E, Weiskrantz L. 1978. Further analysis of the prior learning effect in amnesic patients. Neuropsychologia 16:169-177.

Wixted J. 2004. The psychology and neuroscience of forgetting. Annu Rev Neurosci 55:235-269.

Zhang H, Sachdev P, Wen W, Kochan N, Crawford J, Brodaty H, Slavin M, Reppermund S, Draper B, Zhu W, Kang K, Trollor JN. 2012. Gray matter atrophy patterns of mild cognitive impairment subtypes. J Neurol Sci 315:26-32. 